Proceedings of the 2012 Winter Simulation Conference

C. Laroque, J. Himmelspach, R. Pasupathy, O. Rose, and A. M. Uhrmacher, eds.

\title{
DO THE ATTRIBUTES OF PRODUCTS MATTER FOR SUCCESS IN SOCIAL NETWORK MARKETS?
}

\author{
Paul Ormerod \\ Bassel Tarbush \\ Volterra Partners LLP, London \\ Department of Anthropology, University of Durham \\ U.K. \\ University of Oxford \\ Department of Economics \\ Oxford, U.K. \\ R. Alexander Bentley \\ Dept. of Archaeology \& Anthropology \\ Bristol University \\ Bristol, BS8 1UU, U.K.
}

\begin{abstract}
In social network markets, the act of consumer choice is governed not just by the set of incentives described by conventional consumer demand theory, but by the choices of others in which an individuals payoff is an explicit function of the actions of others. We observe two key empirical features of outcomes in such markets. First, a highly right-skewed, non-Gaussian distribution of the number of times competing alternatives are selected at a point in time. Second, there is turnover in the rankings of popularity over time. We show that such outcomes can arise either when there is no alternative which exhibits inherent superiority in its attributes, or when agents find it very difficult to discern any differences in quality amongst the alternatives which are available so that it is as if no superiority exists. These features appear to obtain, as a reasonable approximation, in many social network markets.
\end{abstract}

\section{INTRODUCTION}

Social network markets are an important and rapidly growing part of the world economy. These are markets in which the act of consumer choice in these industries is governed not just by the set of incentives described by conventional consumer demand theory, but by the choices of others in which an individuals payoff is an explicit function of the actions of others (Potts et al. 2008). Examples include Arthur (1989), De Vany and Walls (1996), Ormerod (1998, 2005, 2007, 2012), and Beck (2007).

Recent social network market models from the network literature have focused on the issues of the dynamics of popularity and the process by which superior alternatives come to the fore A seminal contribution was made by Lieberman, Hauert and Nowak (2005). They used an established evolutionary model to capture the process of selection and choice. They showed that the probability of a new, better invention becoming adopted widely, depends in a fundamental way on the social network structure.

In this paper, we assume instead that either there is no alternative which exhibits inherent superiority in its attributes, or in which agents find it very difficult to discern any differences in quality amongst the alternatives which are available so that it is as if no superiority exists.

We focus our attention on the impact of network structure on two key empirical outcomes of social network markets: first, the right-skewed non-Gaussian distribution of choices amongst alternatives at any point in time. Second, the fact that persistent turnover is observed in the rankings over time.

Ormerod, Tarbush and Bentley (2012) consider four distinct large networks that match those studied by Lieberman et al. (op.cit.): (a) a square lattice, where agents copy other agents near them on a grid, (b) a 


\section{Ormerod, Tarbush, and Bentley}

fully connected network, where each agent can copy any other agent, (c) a "meta-funnel" network, where the structure funnels out from a central agent, and (d) a "superstar" network, where agents are grouped and a central agent is connected to all groups. They find that the key empirical features of social network markets are indeed replicated, using an evolutionary model of behavioral choice. A clear winner emerges without any one of the alternatives exhibiting superiority in its attributes over the others. They suggest that this is a very general principle of social network markets, regardless of whether agents are able to distinguish between the objective attributes of competing alternatives.

In this paper, we focus on three network topologies which are widely observed in social and economic contexts, namely random networks, small world networks and scale-free networks. After a discussion of the key empirical features of social network markets in Section 2, Section 3 describes the behavioral model of agent choice and the network structures examined. Section 4 sets out the results, and section 5 offers a brief discussion and conclusion.

It is important to emphasize from the outset that the model here is based on fixed networks. There is a large amount of evidence that in reality social networks are rarely fixed and more probably dynamic. In some cases, they follow the fact that individuals attach preferably to known partners, create/break their links frequently according to an evaluation of the pertinence/usefulness of the received info, overweigh the opinion of certain friends, opinion makers, and so on. Bravo, Squazzoni and Boero (2012), for example, demonstrate the importance of dynamic networks. This means that it is reasonable to expect that in certain cases individual choices and social networks co-evolve. If this is true, in reality, what is found here with fixed networks could be even more important in the case of networks which evolve dynamically.

\section{SOCIAL NETWORK MARKETS IN PRACTICE}

The music download experiment of Salganik, Dodds and Watts (2006), for example, shows how the impact of social networks dramatically increases the non-Gaussian features of the statistical distribution of the outcome of choice made about different alternatives. The experiment created an artificial 'music market' in which participants downloaded previously unknown songs either with or without knowledge of previous participants' choices. In the experiments with no social influence, in other words with no knowledge of the choices made by others, the participants were asked to rate the quality of the song. Such circumstances offer an approximation to the standard behavioral model of choice in economics, namely that of individuals with fixed tastes and preferences operating in isolation from other agents.

There is only a weak correlation between the market shares obtained by the songs in the experiments which contained social influence, and the quality ratings attached to the songs in the experiments in which the agents acted autonomously. Further, Ormerod (2012) shows that in a typical experiment without social influence, the ratio of the number of downloads of the most popular and least popular songs was approximately $3: 1$. However, in the social influence experiments, the ratio rose typically to around 50:1 (fifty to one).

A slightly irreverent but highly relevant example is given by Godart and Mears (2009). They studied the Style.com show reports for Spring 2007 and found that designers used a total of 677 fashion models worldwide for their shows. Over 75 per cent of the total who appeared, were in 5 shows or less. Only 60 women in the entire modeling universe walked in more than 20 shows, with the market leader of the time, Coco Rocha, being featured in 55 shows. Many aspirants, of course, featured in none. This highly right-skewed outcome was, again, associated with the finding that the attributes of the most successful model was no different to that of the market as a whole.

Simon (1955) conjectured that right-skewed non-Gaussian outcomes were so prevalent in the real world that "its appearance [the non-Gaussian outcome] is so frequent, and the phenomena in which it appears are so diverse, that one is led to the conjecture that if these phenomena have any property in common it can only be a similarity in the underlying probability mechanisms". His examples were: distributions of words in English prose by the frequency of their appearance; distributions of scientists by numbers of 


\section{Ormerod, Tarbush, and Bentley}

papers published; distributions of cities by populations; distributions of incomes by size; distributions of biological genera by numbers of species.

Ormerod (2012) cites a number of other, quite disparate right-skewed non-Gaussian outcomes: downloads on YouTube; film producers' earnings; the number of sexual partners people have; the size of price changes in financial assets; crowds at soccer matches; firm sizes; the size and length of economic recessions; the frequency of different types of endgames in chess; sizes of cities; the ratings of American football coaches in USA Today; the distribution of $£ 1$ million homes across London boroughs; unemployment rates by county in America; deaths in wars; the number of churches per county in William the Conquerors Domesday Book survey of England in the late eleventh century.

The second key empirical feature is that there is turnover in the rankings of popularity over time. This phenomenon occurs on all time scales.

Batty (2006), for example, analyses turnover in the largest cities, in the US and in the world. With the former, over the 1790-2000 period, 266 cities were at some stage in the top hundred. From 1840, when the number of cities first reached one hundred, only twenty-one remain in the top hundred of 2000. On average, it takes 105 years for 50 per cent of cities to appear or disappear from the top hundred, whilst the average change in rank order for a typical city in each ten-year period is seven ranks. In terms of world cities, using data from $430 \mathrm{BC}$, the turnover in the top fifty is slower, but it is still there. Suzhou in China records 2,158 years in the biggest fifty cities of the world, closely followed by Nanking with 2,080 years. No city in the top fifty in 430 BC survives in the list in 2000, but of the fifty biggest at the time of the Fall of Constantinople in 1453, a major event in world history, six are still there.

Almost, but not quite, at the other extreme, Ormerod (2012) notes that over the entire period from 1952 to 2006, no fewer than 29,056 songs appeared in the Top 100 chart in the UK. Of these, 5,141 were in the chart for just a single week. Almost exactly a half stayed in for less than a month, so four weeks was the typical life span, as it were, of a song in the Top 100. In contrast, fifty-nine remained popular for more than six months, and one, 'My Way' by Frank Sinatra, spent an incredible 122 weeks in the chart. The typical life span at number 1 was just two weeks, the longest unbroken reign being sixteen weeks ('(Everything I Do) I Do It For You', by Bryan Adams in 1991).

Turnover in the rankings on internet sites such as YouTube and Flickr is even more rapid. The connection between success and the inherent attributes of the product is tenuous in the extreme. Labash (2012) describes the third biennial ROFL ('rolling on the floor laughing') conference held at MIT. He notes that most of the viral stars believe themselves to be special, that their fame is a reflection of their creativity and individuality. The content, however, suggests that this might not always be the case. For example, 'Keyboard Cat', a video of a cat playing a keyboard in a T-shirt, has registered 25,271,864 views on YouTube. 'Where the Hell is Matt?', in which Matt Harding dances like an idiot in locations across the world, has 43,412,218. And 'Double Rainbow', a video of a man watching a double rainbow, has been viewed 34,458,444 times.

\section{MODEL AND METHODS}

\subsection{The Model}

There is now a stupendous variety of choices available in many consumer markets. example, what Beinhocker (2007), for example, notes that: "The Wal-Mart near JFK Airport has over 100,000 different items in stock, there are over 200 television channels offered on cable TV, Barnes and Noble lists over 8 million titles, the local supermarket has 275 varieties of breakfast cereal, the typical department store offers 150 types of lipstick, and there are over 50,000 restaurants in New York City alone." At the stock keeping unit level (SKU), the level of product detail at which retailers specify their restocking orders, Beinhocker estimates that on a single day in New York, there are 10 billion (!) such choices available. There may indeed be objective differences between the various offers, but in such numerous, minor and often incomprehensible ways that they exemplify what has come to be called 'decision quicksand by Sela and Berger (2011) or 'decision fatigue' by Baumeister and Tierney (2011). 


\section{Ormerod, Tarbush, and Bentley}

Given the huge amount of choice which has emerged in recent decades, the behavioral model of economics, that of rational selection on the basis of objective information, faces challenges, even when it is modified to take into account imperfect and asymmetric information. If rationality is defined as maximizing utility subject to constraints, but every possible good is effectively identical, then every good will be in the argmax of the utility, and therefore every good will be chosen with equal probability.

The evolutionary choice model used here, however, differs from this. As a heuristic in such circumstances, agents essentially choose with a probability equal to the number of times any given alternative has been selected as a proportion of the total number of selections made across the agents to which the agent is connected. They may, for example, regard other agents as having more information than they do, and hence copy their behavior. This is the principle of 'preferential attachment', first articulated by Simon (op.cit.).

The model, and behavioral and psychological evidence supporting the concept, is set out in Ormerod, Tarbush and Bentley (op cit.). We describe here the key features. In essence, the behavioral model is developed from the cultural evolution literature, and has been shown to be capable of explaining empirical outcomes in a wide range of different contexts. A majority of agents in a population copy one another in an effectively undirected manner, and the (small) minority innovate their own original behaviors. A generalization of the model is given in Bentley, Ormerod and Batty (2011).

This modeling approach differs from that based upon the concept of rational addiction with preferences which are learned and are intertemporally dependent (for example, Becker and Murphy 1988, Britto and Barros 2005). In this model, agents are not required to learn preferences over time. Indeed, the preferences of any given agent are not formed over time. At any point in time, an agent makes a choice based simply on the choices made by others, with a small probability of random innovation in making their selection.

The model proceeds as follows: There are $N$ agents in a fixed network. There are initially $N$ possible choices, and we initialize the model with a unique and distinct choice being assigned to each agent. In each period, every agent can make a choice. With probability $1-\mu$ the agent selects a choice with a probability proportional to the number of agents that it is connected to that have made that choice in the previous period, and with probability $\mu$, the agent selects a new choice that is different from all previously available choices. Considerable anthropological and socioeconomic evidence exists (c.f. Ormerod, Tarbush, Bentley, op.cit.) on the plausible values for $\mu$ being no greater than 0.1 .

We ran this model 500 independent times over each of the three types of network structures (approximately 475 nodes), and for 2000 periods each time. The number of agents and lengths of the runs were chosen to be comparable to the arrangements studied in Lieberman, Hauert and Nowak (op.cit.) and in Ormerod, Tarbush and Bentley (op.cit.). We also varied the experimentation parameter $\mu$, allowing it to take the values $0,0.25 \%, 0.5 \%, 0.75 \%, 1 \%$ and $5 \%$.

For each run of the model, we recorded:

- the distribution of choices ranked by popularity

- the life spans of the top 100 longest surviving choices

- the life spans of the top 100 most popular choices (measured by the total number of times the choice was selected over the run of the model)

- the number of choices that are counted among the top 100 longest survivors and the top 100 most popular.

Since the model was run 500 independent times over each network, we obtained the averages over the 500 runs of the above statistics.

\subsection{Network Structures}

We look at Erdos-Renyi, Barabasi-Albert, and Watts-Strogatz network models. All of them are undirected and have 475 nodes. We created three Erdos-Renyi networks that differed only in the parameter $p$, the probability that an edge forms, which was allowed to take the values $0.01,0.025$, and 0.05 . The resulting 


\section{Ormerod, Tarbush, and Bentley}

networks are called ER1, ER2 and ER3, respectively. We created three Barabasi-Albert networks that differed only in the parameter $q$, the number of new edges that are added in each period, which was allowed to take the values 2, 4 and 6 . The resulting networks are called BA1, BA2 and BA3, respectively. (The initial number of nodes was set to $q$ ). We created three Watts-Strogatz networks that differed only in the parameter $r$, the probability of adding a shortcut, which was allowed to take the values $0.25,0.5$, and 0.75 . The resulting networks were called WS1, WS2 and WS3, respectively. (The initial configuration was a simple circle network).

A new network with the specific parameter values was generated in each run of the model. Table 1 sets out summary statistics of the properties of the networks. These are averaged across 500 independent realizations with given parameters.

Table 1: Network statistics: averaged across 500 realisations of each type.

\begin{tabular}{rlllllllll}
\hline & ER1 & ER2 & ER3 & BA1 & BA2 & BA3 & WS1 & WS2 & WS3 \\
\hline Mean degree & 4.7 & 11.9 & 23.7 & 4.0 & 8.0 & 11.9 & 2.5 & 3.0 & 3.5 \\
Median & 4.7 & 11.8 & 23.6 & 2.6 & 5.1 & 8.0 & 2.0 & 3.0 & 3.0 \\
Skewness & 0.5 & 0.3 & 0.2 & 5.8 & 4.4 & 3.7 & 1.1 & 0.8 & 0.7 \\
Kurtosis & 3.2 & 3.1 & 3.5 & 48.8 & 27.7 & 19.5 & 4.1 & 3.6 & 3.7 \\
\hline
\end{tabular}

\section{RESULTS}

The expected number of choices at the end of a model run is approximated by $n(1+\mu(T-1))]$, so for $\mu=0.0025$, for example, there are almost 3,000 alternatives from which to choose whilst with $\mu=0.05$, this number rises to some 50,000. But even with very low values of $\mu$, a large number of choices exist.

Table 2 illustrates the right-skewed nature of the results. This shows the number of times the top ranked (by popularity) alternative is selected after 2000 periods, averaged over 500 runs.

Table 2: The number of times the top ranked (by popularity) alternative is selected after 2000 periods (average over 500 runs).

\begin{tabular}{clllllllll}
\hline$\mu$ & ER1 & ER2 & ER3 & BA1 & BA2 & BA3 & WS1 & WS2 & WS3 \\
\hline 0 & 717163 & 733559 & 730130 & 833679 & 827036 & 822136 & 523143 & 610356 & 649588 \\
0.005 & 91843 & 93828 & 94995 & 130062 & 129763 & 124543 & 55111 & 68267 & 73582 \\
0.01 & 37985 & 38701 & 39212 & 59937 & 55996 & 54835 & 22331 & 27763 & 30922 \\
0.05 & 3199 & 3346 & 3426 & 5810 & 5376 & 5135 & 1859 & 2298 & 2578
\end{tabular}

It is known (Neiman, 1995, Bentley, Ormerod and Batty, 2011) that as the value of the innovation parameter, $\mu$, approaches zero, the outcome approaches a winner-take-all distribution. A small number of alternatives are selected by large numbers of agents, with most choices being selected by few.

Figure 1 shows the resulting rank-size distribution of choices after 2,000 steps of the model for different value of $\mu$, using the Barabasi-Albert network. Comparable figures for the other networks are available on request from the authors We sum the results across all the individual solutions of the model and divide by the total number of solutions. The most frequently selected alternative has rank 1 , the second most frequent rank 2 , and so on.

Figure 2 shows the lifespans of the top 100 longest-surviving choices (where the lifespan of a choice is the number of periods for which the choice is selected by some node, starting from the period at which it is first selected to the period after which no agents select it), this time for the Watts-Strogatz network, and again, comparable figures for the other networks are available on request from the authors. 

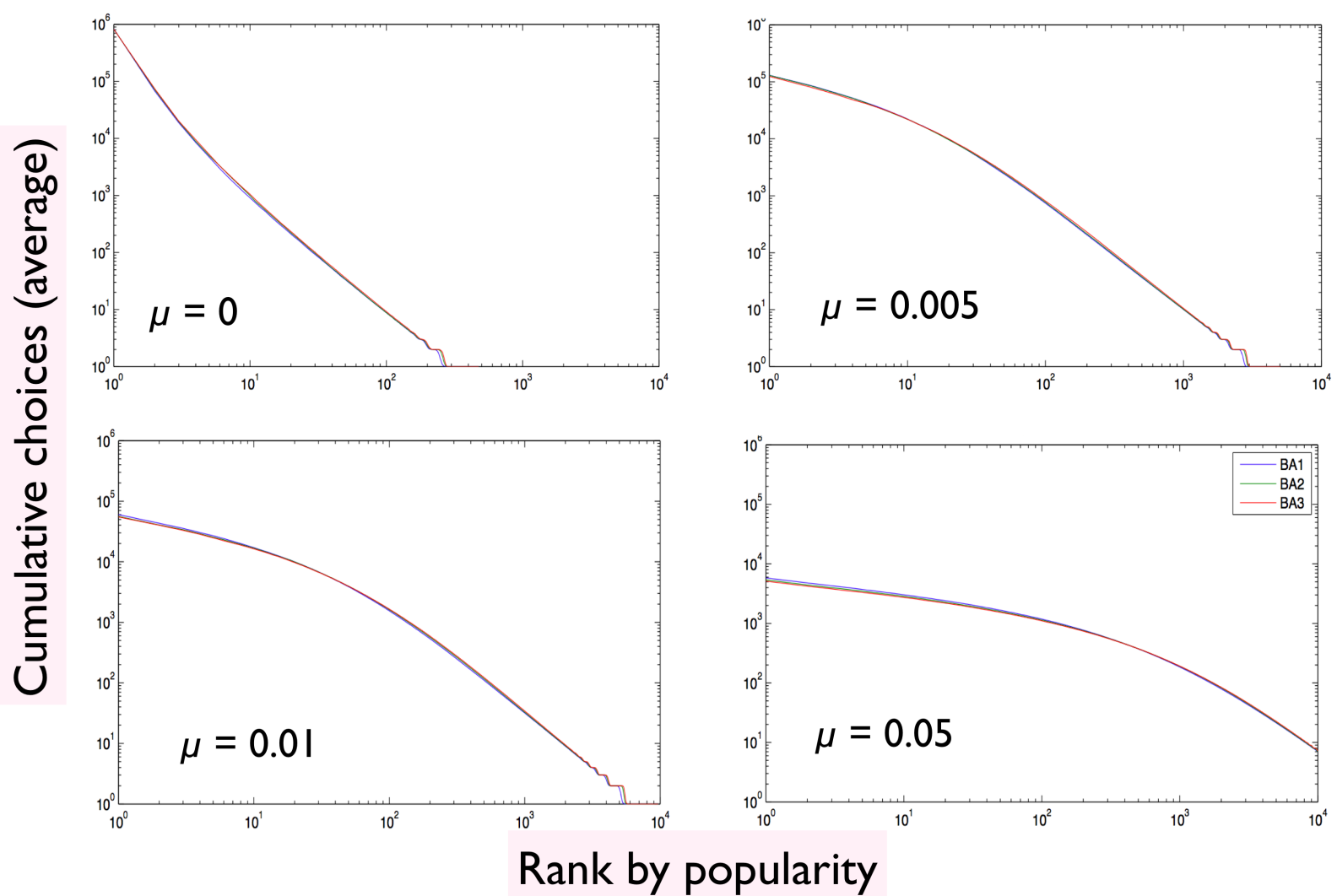

Figure 1: Rank-size distribution of choices after 2,000 steps of the model, for different value of $\mu$, using the Barabasi-Albert network.

\section{DISCUSSION}

It might be imagined that if it is not possible for agents to distinguish between the attributes of alternative choices, so that it is as if no one alternative is superior to any other, that the resulting distribution of the number of times each alternative is selected will be uniformly distributed. However, this is most decidedly not the case. We observe marked right-skewed non-Gaussian outcomes, as in the real world of social network markets, in which decisive 'winners' emerge.

Overall, the results are shaped strongly by the features of the networks. This supports the argument of Foster and Potts (2006) that in social network markets, it is the meso-level structure (i.e. population and system level) which essentially determines the features which are observed at the macro-level (non-Gaussian rank-size distributions, turnover in rankings through time).

\section{CONCLUSION}

We find that that a clear winner emerges in social network markets even when, by assumption, there is no inherent superiority of any of the alternative choices available to agents. Further, there is persistent turnover in the rankings of popularity.

These two key stylized facts of social network markets can be obtained from a model in which, by deliberate assumption, no alternative is superior to any of the others. This may seem a surprising conclusion. However, even a brief acquaintance with the most popular picture on Flickr or the most popular videos on YouTube will, on most days, offer powerful casual empirical support for this conclusion. 


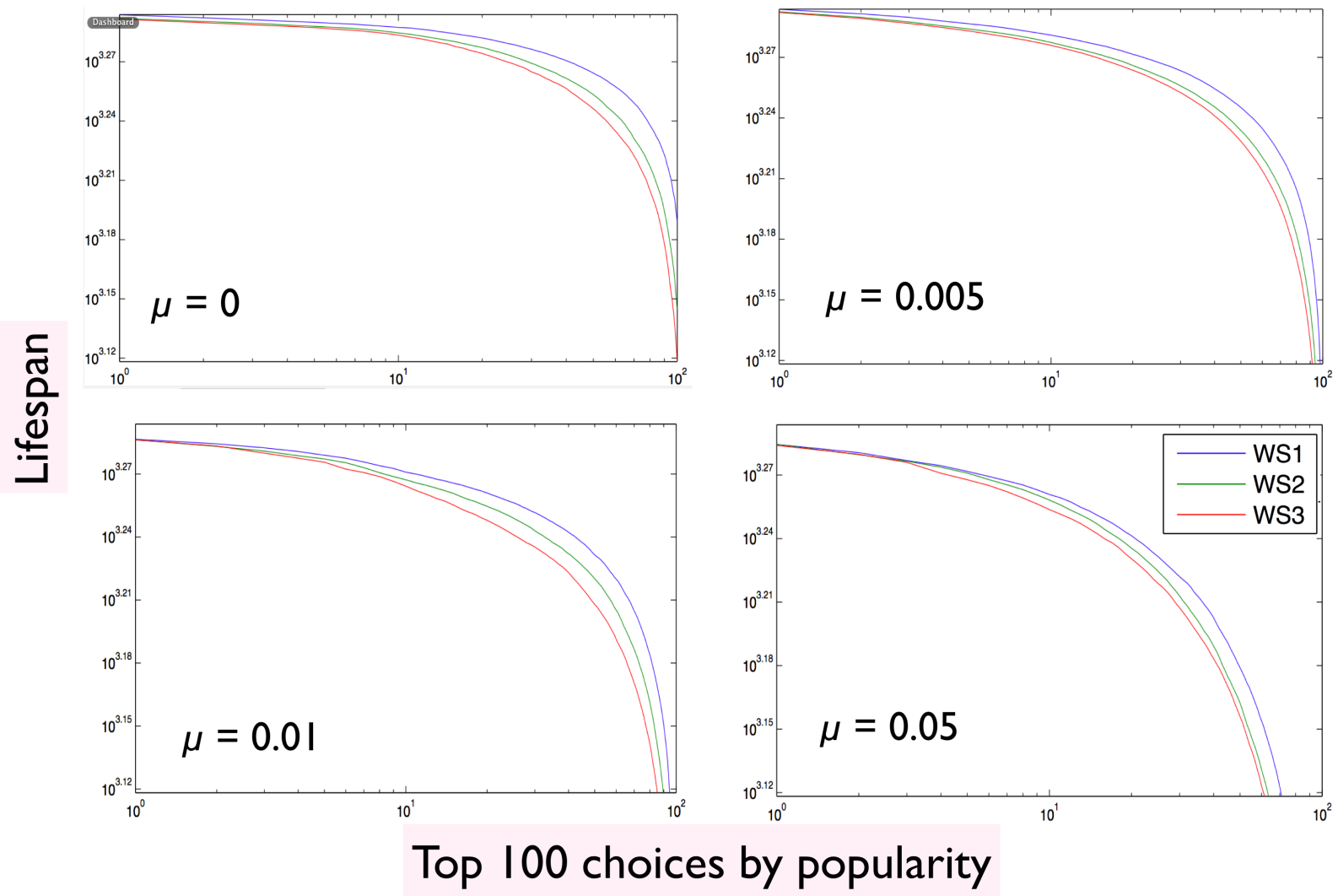

Figure 2: Lifespans of the top 100 longest-surviving choices for the Watts-Strogatz (WS) network.

To emphasize, we find this network effect even in the very restrictive conditions of fixed networks, and with no increasing returns in the model. By this, we mean that if an available alternative becomes popular, under increasing returns it would acquire characteristics which would make it even more popular. In other words, it would seem as if it had superior attributes, not merely that it was popular. By assumption, we rule out increasing returns, and yet we still obtain highly right-skewed non-Gaussian outcomes.

This paper suggests that non-Gaussian outcomes at a point in time and turnover over time seem to be fundamental principles of social network markets. It is a feature of such markets regardless of whether agents are able to distinguish between the objective attributes of competing alternatives.

\section{ACKNOWLEDGEMENTS}

We thank the 'Tipping Points' programme at Durham University, funded by the Leverhulme Trust, for partial support of this project.

\section{REFERENCES}

Arthur, W. B., 1989, “Competing technologies increasing returns and lock-in by historical events. Economic Journal 99: 116-131.

Batty, M., 2006, Rank clocks, Nature, 444: 592-596.

Becker, G. and Murphy, K., 1988, "A theory of rational addiction". Journal of Political Economy 96: 675-700.

Baumeister, R. F., and Tierney, J., 2011, Willpower. New York: Penguin. 


\section{Ormerod, Tarbush, and Bentley}

Beck, J., 2007, "The sale effect of word of mouth: A model for creative goods and estimation for novels". Journal of Cultural Economics 31(1): 5-23.

Beinhocker, E., 2007, The Origin of Wealth. Cambridge, MA: Harvard Business School Press.

Bentley R. A., Ormerod, P., and Batty, M., 2011, "Evolving social influence in large populations". Behavioral Ecology and Sociobiology 65: 537-546.

Bravo G., Squazzoni F., and Boero R., 2012, "Trust and partner selection in dynamic networks: an experimentally grounded model social network". Social Networks, in press

Brito, P., Barros, C., 2005, "Learning by consuming and the dynamics of the demand and prices of cultural goods". Journal of Cultural Economics 29: 83-106.

De Vany, A.and Walls,W. D., 1996, "Bose-Einstein dynamics and adaptive contracting in the motion picture industry”. Economic Journal, Royal Economic Society 106(439): 1493-1514.

De Vany, A. (2004). Hollywood economics. London: Routledge.

Foster, J. and Potts, J., 2006, "Complexity, networks and the importance of demand and consumption in economic evolution." In Flexibility and stability in economic transformation, edited by M. McKelvey and M. Holman, Oxford: Oxford University Press.

Godart, F., and Mears, A., 2009, "How do cultural producers make creative decisions?" Social Forces 88: 671-692.

Labash M., 2012, Memes, supercuts and meta-memes: junk culture or Shakespeare?, The Week, London, 21 July 2012, 48-49.

Lieberman, E., Hauert, C., and Nowak, M. A., 2005, "Evolutionary dynamics on graphs". Nature 433: 312-316.

Neiman, F. D., 1995, "Stylistic variation in evolutionary perspective". American Antiquity 60: 7-36.

Ormerod, P., 1998, Butterfly Economics. London: Faber and Faber.

Ormerod, P., 2005. Why Most Things Fail: Evolution, Extinction and Economics. London: Faber and Faber.

Ormerod, P., 2007, "Extracting deep knowledge from limited information on evolved social networks". Physica A 378: 48-52.

Ormerod, P., 2012, Positive Linking: How Networks Can Revolutionise the World, Faber and Faber London.

Ormerod, P., Tarbush, B, and Bentley, R.A, 2012, "Social network markets: the influence of network structure when consumers face decisions over many similar choices", paper to be presented at the COMPLEX conference, Santa Fe, December 2012, available from the corresponding author, Bentley.

Potts, J., Cunningham, S., Hartley, J., and Ormerod, P., 2008, "Social network markets: a new definition of the creative industries". Journal of Cultural Economics 32(3): 167-185

Salganik, M. J., Dodds, P. S., and Watts, D. J., 2006, "Experimental study of inequality and unpredictability in an artificial cultural market". Science 311: 854-856.

Sela, A., and Berger, J., 2012, "Decision quicksand: how trivial choices suck us in". Journal of Consumer Research 39(2): 360-370.

Simon, H. A., 1955, "A behavioral model of rational choice". Quarterly Journal of Economics 69: 99-118.

\section{AUTHOR BIOGRAPHIES}

PAUL ORMEROD studied economics at Cambridge University and is an economist, best-selling author of Death of Economics, Butterfly Economics, Why Most Things Fail and Positive Linking. He is a partner in Volterra Partners LLP, London. His research interests are networks and complex systems. He has an honorary doctorate from Durham University, UK. His email address is pormerod@ volterra.co.uk.

BASSEL TARBUSH is a Ph.D. student in Economics at the University of Oxford. He received a Bachelor's degree in Economics from Cambridge University. His email address is bassel.tarbush@economics.ox.ac.uk. 


\section{Ormerod, Tarbush, and Bentley}

R. ALEXANDER BENTLEY is Professor of Archaeology and Anthropology at the University of Bristol. His research concerns modeling and understanding collective "herding behavior in society, and testing these models against real-world phenomena. Bentley served as a Program Co-Chair at the $2012 \mathrm{NetSci}$ conference (held at Northwestern University, Evanston IL. His email address is r.a.bentley@bristol.ac.uk. 